Monatsschr Kinderheilkd 2016 $\cdot 164: 89-90$ DOI 10.1007/s00112-015-0028-0

Online publiziert: 19. Januar 2016

๑) Springer-Verlag Berlin Heidelberg 2015

CrossMark

\author{
J. Dötsch ${ }^{1} \cdot$ F. Zepp ${ }^{2}$ \\ ${ }^{1}$ Klinik und Poliklinik für Kinder- und Jugendmedizin, Universitätsklinikum Köln, Köln, Deutschland \\ ${ }^{2}$ Kinderklinik und Kinderpoliklinik, Johannes-Gutenberg-Universität, Mainz, Deutschland
}

\title{
Fetale Programmierung und frühkindliche Prägung
}

Das Konzept ist ebenso einprägsam wie verlockend: In einer frühen Phase des Lebens, d. h. vor allen Dingen in der intrauterinen Zeit und in den ersten zwei Lebensjahren werden durch Umwelteinflüsse im weiteren Sinne vegetative Prozesse festgelegt, die für Gesundheit und Lebensqualität im Verlauf des weiteren Lebens von entscheidender Bedeutung sind.

Die Folgen eines gestörten intrauterinen Milieus oder einer frühen postnatalen vergleichbaren Situation sind vor allem in den zahlreichen Zivilisationskrankheiten wie Adipositas, Herz-Kreislauf-Erkrankungen, aber auch psychiatrischen und malignen Erkrankungen wiederzufinden.

Eine Reihe von Problemen ist allerdings sowohl mit dem Konzept selbst als auch mit dem Forschungsfeld verbunden. Zum einen die verlockend einfache Vorstellung, dass ein Fetus im Mutterleib eine Situation erlernt, die ihn für das spätere Leben vorbereitet. Zum Beispiel dadurch, dass der Mangel an Nährstoffen in einem Mangelgebiet besser für das postnatale Leben befähigt. Kritisch wird diese Vorstellung besonders dann, wenn hier teleologisch, d. h. unter der Vorstellung, die Natur habe sich „etwas dabei gedacht", argumentiert wird. Nicht nur, dass eine solche Vorstellung evolutionsbiologischen Vorgängen widerspricht, sie birgt auch die Gefahr, die empirische Vorgehensweise zu verlassen und damit ein Forschungsgebiet potenziell populärwissenschaftlich zu diskreditieren.

Ein zweites kritisches Feld im Gebiet der perinatalen Programmierung und frühkindlichen Prägung ist der Umstand, dass im Gegensatz zu genetischen Vorgängen in der Regel kein früher Phänotyp zu finden ist. Der Phänotyp entsteht erst durch die Einwirkung weiterer Faktoren. Das klassische Beispiel hierfür ist die Adipositas, eine von wenigen genetischen Ausnahmen abgesehen multifaktoriell bedingte Erkrankung. Lebensgewohnheiten wie Rauchen und andere im Laufe des Lebens zugeführte Toxine können darüber hinaus Herz-Kreislauf-Erkrankungen und Malignomentstehung in einer Weise beeinflussen, dass die Effekte der perinatalen Programmierung nicht mehr sichtbar sind.

\section{》) Frühkindliche Prägung ist ein relevantes Forschungsfeld der Pädiatrie}

Im Kontext einer biologisch zunehmend mechanistisch ausgerichteten Forschungslandschaft stellt sich schließlich die Frage nach den exakten Mechanismen. Epigenetische, d. h. das Erbgut sekundär beeinflussende, Veränderungen konnten häufig nachgewiesen werden, sind jedoch in vielen Fällen eher als Surrogat oder Indiz zu betrachten und weniger als kausalmechanistische Erklärung. Insofern gestaltet sich ein hochinteressantes Feld mit enormer Relevanz für die Pädiatrie und den Übergang ins Erwachsenenalter an verschiedenen Punkten noch entwicklungsfähig.

Das vorliegende Heft fasst den aktuellen Stand, die Konzepte und die möglichen zukünftigen Implikationen auf dem Gebiet der frühkindlichen Prägung für den Kinder- und Jugendarzt zusammen.

Wesentliche konzeptionelle Momente und die Frage der Einordnung der perinatalen Programmierung in unser derzeitiges Wissen von biologischen Abläu- 
fen werden durch einen der Pioniere auf dem Gebiet, Herrn Prof. Plagemann aus Berlin, dargestellt.

Klinisch und wissenschaftlich tätige ärztliche Experten auf dem Gebiet der perinatalen Programmierung, namentlich Frau Prof. Ensenauer, Frau Dr. Hucklenbruch-Rother und Frau Dr. Brüll sowie Herr Dr. Dr. Alcazar und Herr und Frau Dr. Nüsken, adressieren die beiden wichtigsten und häufigsten Formen der Störung des intrauterinen Milieus, nämlich die intrauterine Überversorgung und die intrauterine Unterversorgung. Sie zeigen neben klinisch relevanten Aspekten die aktuellen Forschungsansätze in diesem Gebiet auf.

Den Abschluss bildet die Betrachtung der postnatalen Phase durch einen der wesentlichen Protagonisten der Säuglingsernährungsforschung, Herrn Prof. Koletzko, insbesondere die Frage der Eiweißzufuhr hat er gemeinsam mit seinen Co-Autoren in seinem Beitrag dargestellt.

Wir hoffen, mit dieser Zusammenstellung für Sie, liebe Kolleginnen und Kollegen, als praktisch tätige Kinder- und Jugendärztinnen und -ärzte einen umfassenden Einblick in ein wesentliches, noch relativ neues Feld der Pädiatrie geben zu können, von dem wir uns in der Zukunft viele neue Ansätze für frühe Prävention versprechen und das damit nicht nur politisch sehr im Trend der Zeit liegt, sondern auch noch einmal eine neue Rolle der Kinder- und Jugendmedizin im Gesamtkontext der Ärzteschaft bilden könnte.

In diesem Sinne wünschen wir Ihnen viel Vergnügen und neue Erkenntnisse bei der Lektüre.

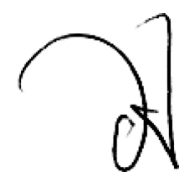

Prof. Dr. J. Dötsch

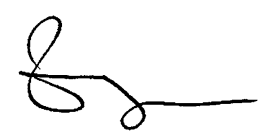

Prof. Dr. F. Zepp

\section{Korrespondenzadresse}

\section{Prof. Dr. J. Dötsch}

Klinik und Poliklinik für Kinder- und Jugendmedizin, Universitätsklinikum Köln Kerpener Str. 62, 50937 Köln, Deutschland joerg.doetsch@uk-koeln.de

Interessenkonflikt. J. Dötsch und F. Zepp geben an, dass kein Interessenkonflikt besteht.
Der Beitrag „Koinfektionen mit humanem Immundefizienzvirus und Tuberkulose" (Maritz ER, Schimana W 2015, Monatsschr Kinderheilkd 163:1130-1137) enthält eine falsche Angabe zur Anzahl der TB-Neuinfektionen unter Kindern weltweit im Jahr 2013. Diese liegt gemäß dem WHO Global Tuberculosis Report 2014 bei 550.000 . Die erwähnte Zahl von 80.000 bezieht sich auf die Anzahl der Todesfälle durch TB bei HIV-negativen Kindern.

Wir bitten den Fehler zu entschuldigen. 